18

\title{
Оптические эффекты в магнитоплазмонных кристаллах на основе 1D металл-диэлектрической решетки
}

\author{
() В.Б. Новиков ${ }^{1}$, А.М. Ромашкина ${ }^{1}$, Д.А. Езенкова ${ }^{2,3}$, И.А. Родионов ${ }^{2,3}$, К.Н. Афранасьев ${ }^{2,4}$, \\ А.В. Барышев ${ }^{2}$, Т.В. Мурзина ${ }^{1, \uparrow}$ \\ ${ }^{1}$ Физический фракультет МгУ им. М.В. Ломоносова, \\ 119991 Москва, Россия \\ ${ }^{2}$ Всероссийский научно-исследовательский институт автоматики им. Н.Л. Духова, \\ 127055 Москва, Россия \\ ${ }^{3}$ Московский государственный технический университет им. Н.Э. Баумана, \\ 105005 Москва, Россия \\ ${ }^{4}$ Институт теоретической и прикладной электродинамики РАН, \\ 125412 Москва, Россия \\ Ie-mail: murzina@mail.ru \\ Поступила в редакцию 15.03.2020 г. \\ В окончательной редакции 15.03.2020 г. \\ Принята к публикации 20.05.2020 г.
}

Методами оптической и магнитооптической спектроскопии исследованы свойства одномерных пространственно-периодических металлических структур, образованных диэлектрической решеткой с нанесенными на нее пленками золота и пермаллоя. Показано, что в таких структурах наблюдается возбуждение поверхностных плазмон-поляритонов, резонансной частотой которых можно управлять выбором геометрии эксперимента. В спектральной окрестности плазмонного резонанса наблюдается модуляция магнитооптического эффекта Керра, отсутствующая в случае непрофилированной бипленки золото/пермаллой.

Ключевые слова: поверхностные плазмон-поляритоны, магнитооптический эффект Керра.

DOI: $10.21883 /$ OS.2020.09.49878.98-20

\section{1. Введение}

Задача усиления оптических эффектов занимает в фотонике центральное место. Перспективным методом ее решения является структурирование материалов на микромасштабах, сопровождающееся возникновением оптических резонансов, обусловленных геометрией структур, в требуемом оптическом диапазоне [1]. Дополнительные функциональные возможности возникают в случае, если микроструктуры образованы, в том числе магнитным материалом, что, как было показано в ряде работ, позволяет значительно повысить эффективность обычно слабых магнитооптических эффектов. Так, было продемонстрировано усиление фарадеевского вращения в одномерных фотонных кристаллах и микрорезонаторах на основе магнитного диэлектрика [2,3]. Интерференционная природа формирования оптических свойств таких фотонных систем принципиально требует их достаточно больших размеров, составляющих в направлении периодичности единицы длин волн. Это ограничение можно преодолеть с помощью наноструктур, собственными электромагнитными модами которых являются поверхностные плазмон-поляритоны (ППП) - поверхностные волны, распространяющиеся вдоль границы раздела металла и диэлектрика [1]. В таких наноструктурах было продемонстрировано резонансное усиление амплитуды электромагнитного поля в субволновой окрестности поверхности металла, приводящее к усилению комбинационного рассеяния света [4], а при наличии ферромагнитного магнитного материала - к усилению магнитооптического эффекта Керра [5,6].

Длина волнового вектора поверхностного плазмонполяритона $k_{\mathrm{spp}}$ определяется диэлектрическими проницаемостями граничащих веществ [7]:

$$
k_{\mathrm{spp}}=k_{0} \sqrt{\frac{\varepsilon_{M} \varepsilon_{d}}{\varepsilon_{M}+\varepsilon_{d}}},
$$

где $\varepsilon_{M}$ и $\varepsilon_{d}-$ диэлектрические проницаемости металла и диэлектрика соответственно, и превышает величину волнового вектора световой волны в свободном пространстве $k_{0}$. В результате излучение, падающее на гладкую металлическую поверхность, не может напрямую возбудить ППП. Поэтому для исследования ППП используются специальные экспериментальные схемы.

Периодическое структурирование металлических пленок, т. е. формирование плазмонных кристаллов, позволяет возбуждать ППП за счет дифракции падающего излучения. В этом случае удается выполнить условия квазисинхронизма

$$
\mathbf{k}_{\mathrm{spp}}=\mathbf{k}_{\|}+m \mathbf{G}
$$

между тангенциальной компонентой волнового вектоpa $\mathbf{k}_{\|}$волны, падающей на плазмонный кристалл, и вектором ППП путем вовлечения вектора обратной решетки периодической структуры $\mathbf{G}$, где $m-$ порядок 
дифракции. Такой дифракционный подход позволяет избежать применения призменных схем возбуждения ППП (Кречмана [8] и Отто [9]), т. е. создавать миниатюрные фотонные ППП-устройства.

Плазмонные кристаллы, в состав которых входят магнитные материалы, принято называть магнитными плазмонными кристаллами (МПК) [10-13]. Несмотря на то, что использование ферромагнитных металлов, как правило, сопровождается повышением оптических потерь в плазмонных кристаллах, удается получить высокодобротные плазмонные резонансы при использовании магнитных диэлектриков (феррит-гранатов) и благородных металлов (золото, серебро), демонстрирующих минимальные потери в видимом спектральном диапазоне по сравнению с другими металлами [14]. В таких структурах достигнуто усиление магнитооптических эффектов более чем на порядок по сравнению с неструктурированным магнитным материалом $[12,13]$. Резонансное поведение магнитооптических эффектов в МПК во многом связано с зависимостью закона дисперсии ППП от приложенного магнитного поля. В частности, для границы раздела металл/магнитный диэлектрик при ориентации намагниченности М последнего в плоскости интерфейса и перпендикулярно направлению распространения ППП, закон дисперсии этой поверхностной волны приобретает вид [15]:

$$
k_{\mathrm{spp}}=k_{0} \sqrt{\frac{\varepsilon_{M} \varepsilon_{d}}{\varepsilon_{M}+\varepsilon_{d}}}(1+\beta g),
$$

где $g \sim M$ - длина вектора гирации для намагниченного диэлектрика и $\beta=\left(-\varepsilon_{M} \varepsilon_{d}\right)^{-1 / 2}\left(1-\varepsilon_{d}^{2} / \varepsilon_{M}^{2}\right)^{-1}$. В результате приложение магнитного поля приводит к угловому или спектральному сдвигу резонанса ППП в МПК. Нечетное по отношению к внешнему магнитному полю смещение резонансов приводит к появлению магнитооптического интенсивностного эффекта в прошедшем или отраженном излучении, который можно описывать путем введения магнитного контраста относительного изменения интенсивности пропускания (отражения) I для противоположных направлений намагниченности, $\pm M$

$$
\rho=\frac{I(+M)-I(-M)}{I(+M)+I(-M)} .
$$

Было показано, что в случае МПК в спектральной окрестности возбуждения ППП усиление линейных магнитооптических явлений может достигать десятков процентов. Этот эффект в основном определяется оптическими свойствами материалов, поддерживающих распространение ППП, и величиной намагниченности на их границе.

Альтернативный подход к созданию МПК основан на применении пленок ферромагнитного металла вместо магнитного диэлектрика. Такие материалы проявляют высочайшую магнитооптическую активность, что важно для создания управляемых магнитным полем устройств фотоники. Поэтому внимание обращено к гибридным магнитоплазмонным структурам, в которых благородный металл поддерживает плазмонные резонансы высокой добротности и магнитооптические эффекты, обеспеченные добавлением ферромагнитного металла, усиливаются [16,17].

В контексте данной тематики перспективным представляется поиск и изучение новых видов МПКструктур. В данной работе предложена структура на основе $1 D$ диэлектрической решетки, на которую последовательно осаждены слои золота и ферромагнитного металла (пермаллоя). Показано, что при толщине пермаллоя $10 \mathrm{~nm}$ такая структура работает как МПК, а величина магнитного эффекта Керра в области резонансного возбуждения ППП превышает значения этого магнитооптического эффекта от неструктурированной пленки пермаллоя.

\section{2. Методика эксперимента}

Исследуемые плазмонные кристаллы были изготовлены с использованием методики, развитой для плазмонных кристаллов на основе золота, нанесенного поверх структурированного диэлектрика [18]. Они представляли собой двухслойную пленку из золота толщиной $80 \mathrm{~nm}$ и пермаллоя $\left(\mathrm{Ni}_{80} \mathrm{Fe}_{20}\right)$ толщиной $10 \mathrm{~nm}$, последовательно нанесенных на одномерную диэлектрическую дифракционную решетку с периодом $650 \mathrm{~nm}$. Решетка была получена методом электронной литографии. В качестве резиста был использован водородный силсесквиоксан (HSQ), высота полосок из этого полимера составляла $63 \pm 5 \mathrm{~nm}$; в качестве подложки был использован плавленый кварц (UV-Grade). Для лучшей адгезии золота на поверхность диэлектрической решетки наносился слой титана толщиной 6-7 nm. Использование золота в изучаемой пространственно-периодической структуре обеспечивает возбуждение плазмонных резонансов достаточно высокой добротности, а наличие слоя ферромагнитного металла обеспечивает выраженные магнитооптические свойства. Изображение структуры, полученное с помощью растрового электронного микроскопа (РЭМ), приведено на рис. $1, b$. Методами магнитометрии и магнитооптического эффекта Керра показано, что насыщение намагниченности структуры достигается в магнитном поле с напряженностью менее $1 \mathrm{kOe}$.

Отметим, что при толщине (сплошной) пленки золота $80 \mathrm{~nm}$ с нанесенным поверх нее слоем пермаллоя $10 \mathrm{~nm}$ структура была практически непрозрачна в видимом диапазоне спектра, поэтому экспериментальные исследования проводились в геометрии на отражение света.

Изучение линейных магнитооптических эффектов проводилось методом спектрокопии отражения при угле падения зондирующего излучения $22.5^{\circ}$, источником которого являлась галогенная лампа. Коллимированный пучок зеркально отраженного света регистрировался 

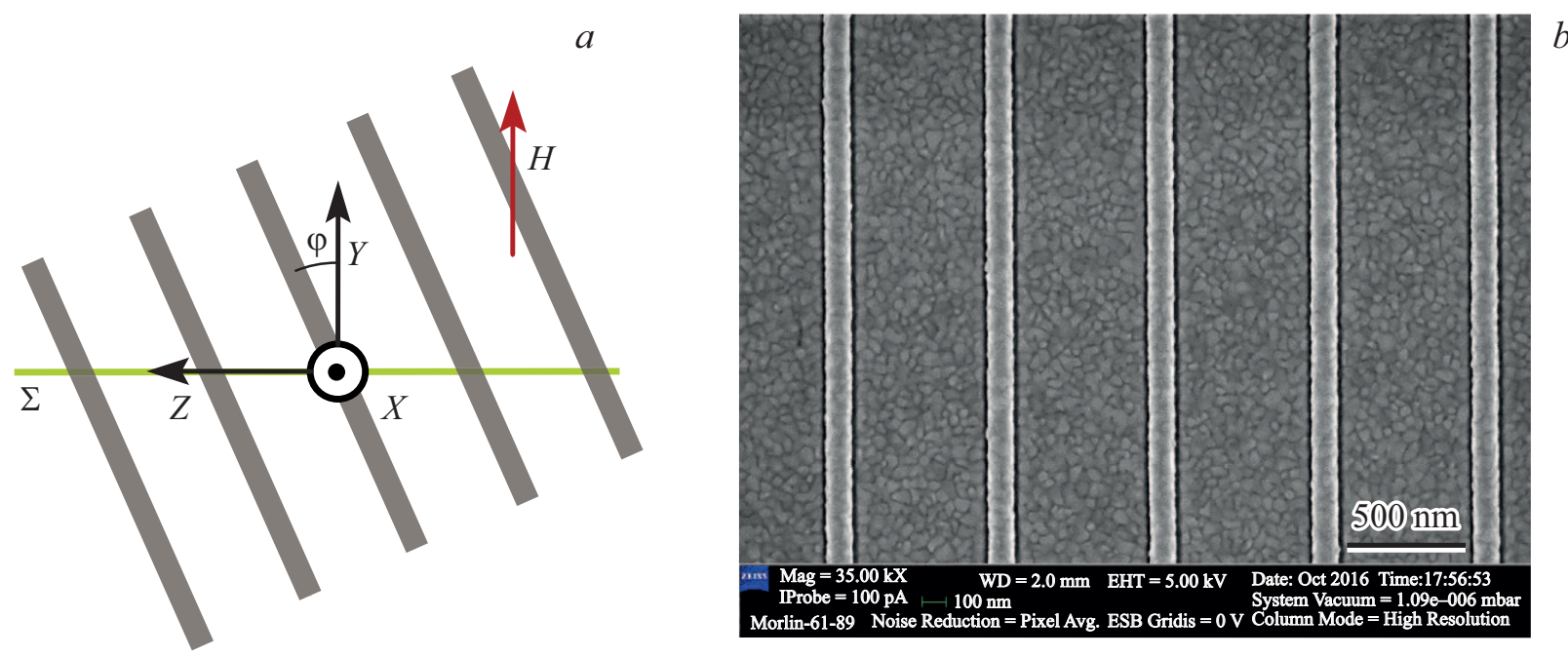

Рис. 1. (a) Схема геометрии эксперимента и одномерной решетки в лабораторной системе координат. $H-$ внешнее магнитное поле, $\varphi$ - азимутальный угол поворота структуры, $\Sigma$ - плоскость падения света; $(b)$ РЭМ-изображение структуры после напыления золота.

спектрометром в диапазоне длин волн от $365 \mathrm{~nm}$ до $1000 \mathrm{~nm}$. Для подстройки резонансной длины волны ППП была реализована возможность азимутального поворота образца (вокруг нормали к своей поверхности); для этого он был помещен на вращающийся столик, обеспечивающий диапазон азимутальных углов $\varphi= \pm 100^{\circ}$; нулевое значение угла $\varphi$ соответствовало случаю, когда направление периодичности структуры параллельно оси $O Z$, т.е. лежало в плоскости падения света. Схематичное изображение образца с указанием лабораторной системы координат, азимутального угла $\varphi$ и направления приложенного статического магнитного поля Н приведено на рис. 1, $a$. Все спектры отражения были нормированы на спектры коэффициента отражения однородной (неструктурированной) бислойной пленки золото+пермаллой, нанесенных на плоскую подложку в аналогичных условиях.

Магнитооптические эффекты в описанной выше структуре были исследованы в геометрии экваториального магнитооптического эффекта Керра. Образец был закреплен между полюсами двух постоянных неодимовых магнитов, создающих постоянное магнитное поле с напряженностью $3 \mathrm{kOe}$, ориентированное перпендикулярно плоскости падения света. Смена направления магнитного поля осуществлялась поворотом жестко закрепленной пары магнитов на $180^{\circ}$, что позволяло измерить магнитный контраст коэффициента отражения света, определяемый формулой (4). Значения $\rho$, приведенные на графиках ниже, были получены путем многократного повторения измерений в положительном и отрицательном направлениях внешнего магнитного поля и последующего усреднения данных.

\section{3. Экспериментальные результаты}

Спектры коэффициента отражения описанных выше пространственно-периодических структур были измерены для $p$ - и $s$-поляризованного зондирующего света для диапазона длин волн от $500 \mathrm{~nm}$ до $1000 \mathrm{~nm}$. На рис. 2, $a$ приведены спектральные зависимости, полученные в случае $p$-поляризованного падающего излучения. Разными цветами отмечены кривые, полученные для значений азимутального угла $0^{\circ}, 10^{\circ}, 20^{\circ}$ и $30^{\circ}$ для $p$-поляризации падающего света. Можно отметить две особенности этих спектров, проявляющиеся благодаря пространственнопериодической структуризации подложки: (i) наличие минимума отражения при длинах волн менее $600 \mathrm{~nm}$, спектральное положение которого не зависит от азимутального угла структуры; (ii) наличие минимума в окрестности длины волны $900 \mathrm{~nm}$, положение которого смещается в сторону коротких длин волн с ростом азимутального угла. На вставке к рис. 2, a приведена экспериментальная зависимость спектрального положения длинноволнового минимума от азимутального положения образца $\varphi$. Видно, что изменение угла $\varphi$ от нуля до $30^{\circ}$ приводит к смещению минимума отражения приблизительно на $50 \mathrm{~nm}$.

В случае $s$-поляризации пробного излучения спектральные зависимости коэффициента отражения имеют более сложную форму (рис. 3,a), соответствующие измерения были выполнены для больших значений азимутального угла $\varphi=60^{\circ}, 70^{\circ}, 80^{\circ}$ и $90^{\circ}$. Аналогично случаю $p$-поляризации, наблюдается минимум в коротковолновой области спектра. Минимумы отражения, отсутствующие в случае бислойной пленки золото/пермаллой на плоской подложке, при длинах волн свыше $600 \mathrm{~nm}$ также смещаются в коротковолновую область спектра при увеличении $\varphi$. Зависимость длины волны минимума 

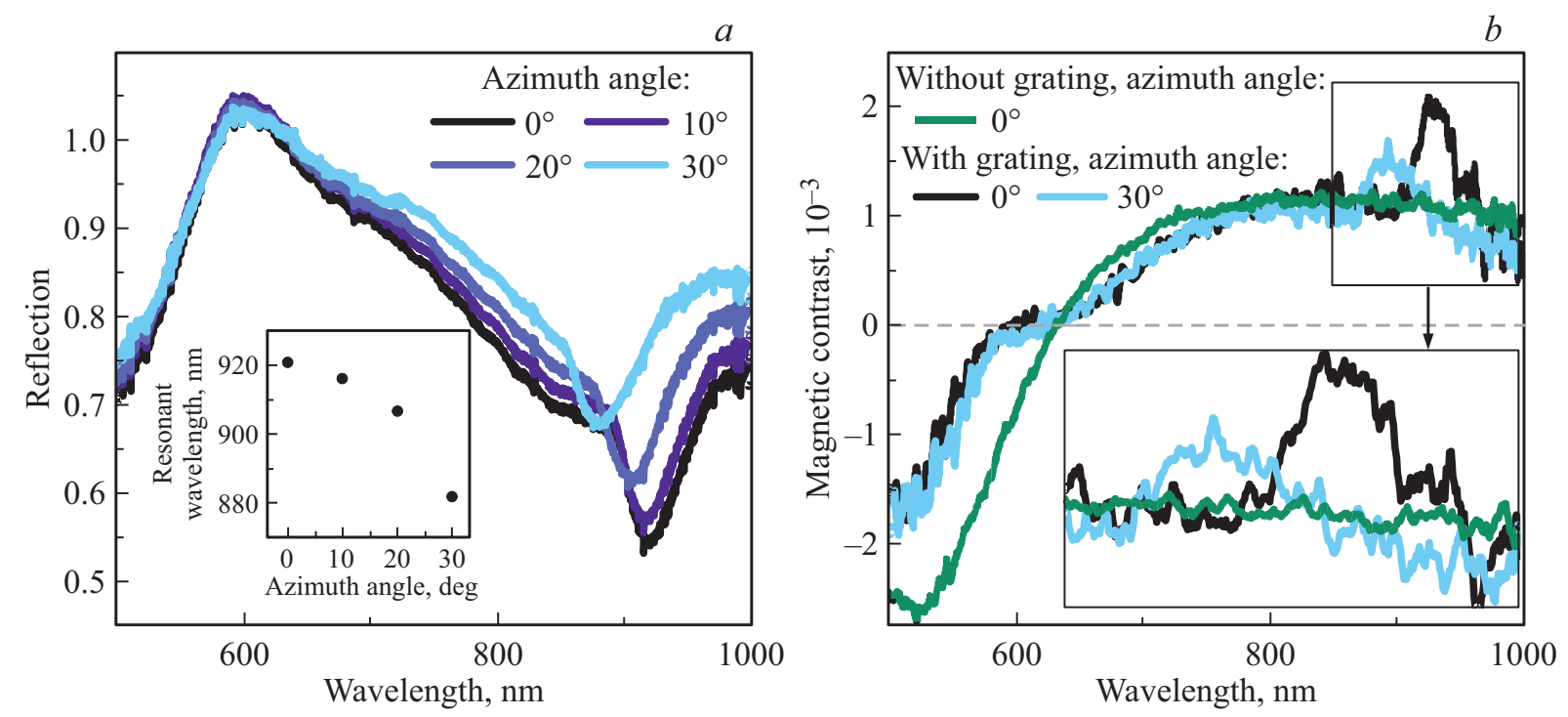

Рис. 2. (a) Спектры коэффициента отражения света при различных азимутальных углах для $p$-поляризованного излучения, на врезке азимутальная зависимость резонансной длины волны минимума коэффициента отражения; $(b)$ спектр магнитного контраста при различных азимутальных углах для $p$-поляризованного излучения.

отражения от угла $\varphi$ приведена на вставке к рис. $3, a$. Спектры магнитного контраста коэффициента отражения исследованных МПК для $p$ - и $s$-поляризованного пробного излучения приведены на рис. $2, b$ и рис. $3, b$ соответственно. Отметим, что магнитный контраст для неструктурированной пленки обращается в ноль в случаe $s$-поляризации света, а при $p$-поляризации меняет знак в окрестности длины волны $650 \mathrm{~nm}$ (рис. $3, b$ ).

Спектры магнитного контраста, полученные для МПК и референсной бислойной пленки, напыленной на плоскую подложку, для $p$-поляризации демонстрируют качественно схожее поведение и смену знака $\rho$ вблизи длины волны $650 \mathrm{~nm}$. Различие магнитооптических свойств МПК и бипленки проявляется в окрестности минимумов коэффициента отражения света от МПК. Плазмонная структура демонстрирует усиление магнитного контраста приблизительно в 2 раза по сравнению со случаем однородной пленки пермаллоя. При этом максимумы магнитного контраста при изменении азимутального угла смещаются вслед за минимумами отражения (рис. 2).

Как было отмечено выше, магнитный контраст для отраженного излучения отсутствует в случае бислойной пленки, помещенной на плоской подложке, при $s$-поляризации пробного излучения. В то же время, для МПК в $s$-поляризованном свете наблюдается знакопеременная спектральная зависимость $\rho$ (рис. $3, b$ ). Сложная форма наблюдаемого спектра магнитного контраста обусловлена одновременным возбуждением двух плазмон-поляритонов, распространяющихся в разных направлениях по отношению к магнитному полю, при азимутальном положении образца $\varphi=90^{\circ}$.

\section{4. Обсуждение результатов}

При анализе полученных результатов рассмотрим вначале возможные механизмы особенностей, наблюдающихся в спектрах линейного отражения. Поскольку в нашей работе управление резонансным спектром МПК осуществлялось за счет азимутального поворота структуры при неизменном угле падения, то смещение спектральных особенностей при изменении азимутального угла $\varphi$ указывало на их связь с возбуждением поверхностных плазмонов в структуре. Таким образом, длинноволновые минимумы спектральных зависимостей коэффициента отражения вызваны резонансным возбуждением ППП в периодической структуре, а их частоты (длины волн) определяются из соотношения (2).

При отклонении азимутального угла $\varphi$ от нуля вектор обратной решетки уже не лежит в плоскости падения света, вызывая изменение длины результирующего вектора $k_{\|} \mathbf{e}_{x}+m \mathbf{G}$, что приводит к смещению резонансной частоты возбуждения ППП. Именно этот эффект и наблюдается как смещение ярко выраженного минимума в спектрах отражения $p$-поляризованного излучения при изменении угла $\varphi$. Соответствие данной спектральной особенности возбуждению ППП первого порядка подтверждается также выполненными расчетами, в которых были взяты табличные значения для показателей преломления материалов МПК в рассматриваемом диапазоне длин волн.

Из рис. 2, $a$ также следует, что увеличение азимутального угла $\varphi$ от $0^{\circ}$ до $30^{\circ}$ приводит к возрастанию коэффициента отражения для $p$-поляризованного падающего света, в то время как глубина модуляции минимума, связанного с ППП, несколько уменьшается. Это связа- 

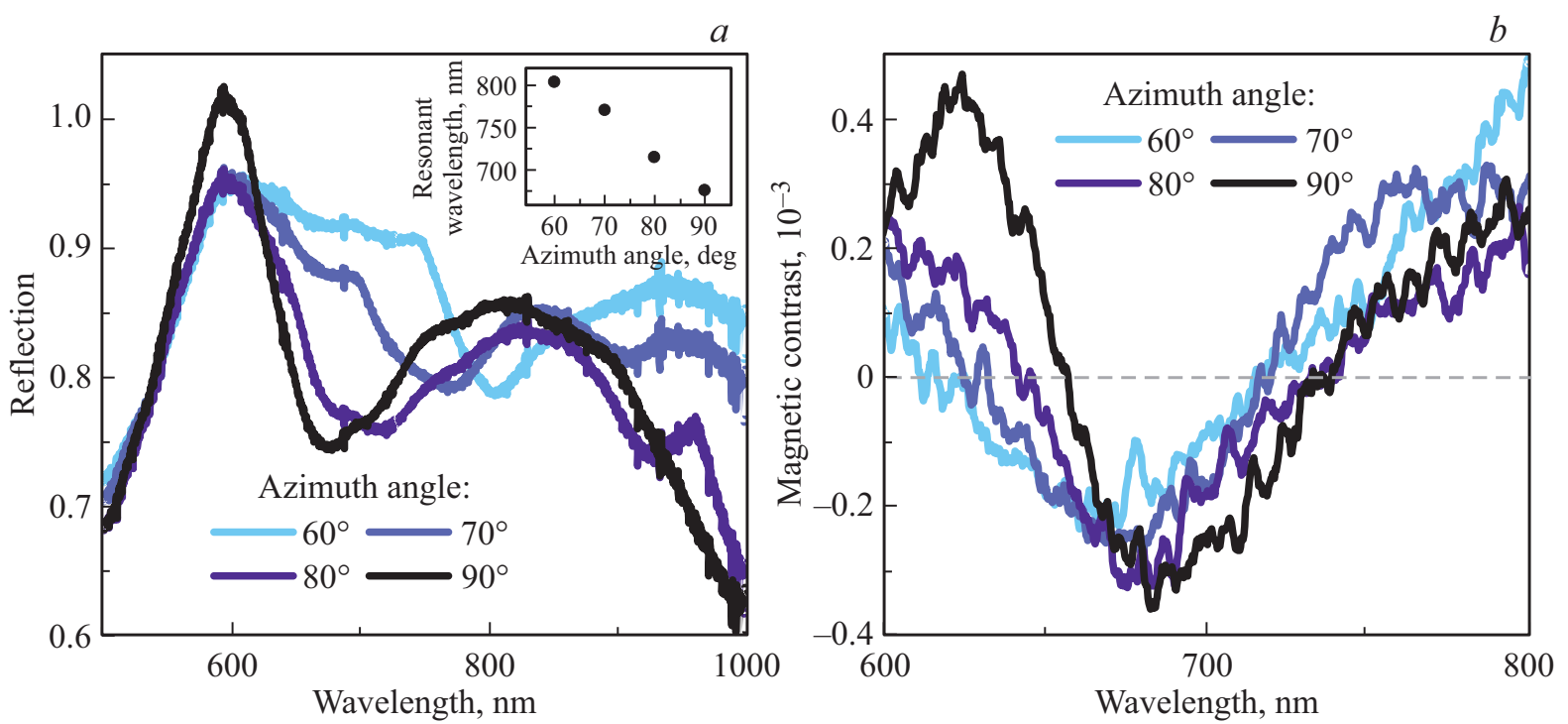

Рис. 3. (a) Спектры коэффициента отражения света при различных азимутальных углах для $s$-поляризованного излучения, на врезке азимутальная зависимость резонансной длины волны минимума коэффициента отражения; $(b)$ спектр магнитного контраста при различных азимутальных углах для $s$-поляризованного излучения.

но с уменьшением эффективности возбуждения ППП при увеличении $\varphi$, которое в свою очередь вызвано пространственным рассогласованием направлений векторов напряженности электрического поля ППП $\mathbf{E}_{\mathrm{spp}}$ и падающей $p$-поляризованной волны $\mathbf{E}_{p}$. Действительно, при увеличении $\varphi$ и приближении его значения к $90^{\circ}$ скалярное произведение $\mathbf{E}_{\mathrm{spp}} \cdot \mathbf{E}_{p}$ обращается в ноль поля ППП и падающей волны становятся ортогональны. Наилучшее согласование полей $\mathbf{E}_{\mathrm{spp}}$ и $\mathbf{E}_{p}$ имеет место при $\varphi=0^{\circ}$, приводя к наибольшей эффективности возбуждения ППП и наиболее глубокому минимуму в спектре отражения света от МПК (рис. 2, $a$ ).

Спектр магнитного контраста для $p$-поляризации зондирующего излучения демонстрирует практически симметричные спектральные максимумы, центральная частота которых соответствует резонансному возбуждению ППП. Наибольшее усиление магнито-оптического эффекта наблюдается при $\varphi=0^{\circ}$ и соответствует максимальной добротности ППП. Усиление магнитного контраста в данном случае связано, по-видимому, с уменьшением интенсивности отраженного излучения, тогда как величина магнитооптической компоненты отклика остается неизменной.

Для $s$-поляризованного падающего света, наоборот, эффективность возбуждения ППП максимальна при азимутальном угле $90^{\circ}$, когда вектор электрического поля зондирующего излучения $\mathbf{E}_{s}$ параллелен направлению распространения волны поверхностного плазмона $\mathbf{E}_{\mathrm{spp}}$ (рис. 3). Отклонение от такой ориентации структуры приводит к достаточно сложному виду спектра отражения, доминирующим вкладом в который является смещение минимума в длинноволновую область спектра. Эта особенность, противоположная направлению спектрального смещения минимумов для $p$-поляризации света при вариации $\varphi$, согласуется с законом дисперсии поверхностного плазмона в периодической структуре, даваемому соотношением (2). В то же время следует отметить, что при (азимутальной) ориентации МПК под углом $\varphi$, близким к $90^{\circ}$, резонансные условия возбуждения ППП выполняются одновременно для двух видов ППП, распространяющихся под разными углами. При отклонении $\varphi$ меняются значения резонансных длин волн и амплитуды соответствующих плазмонных мод. Так, эффективность возбуждения ППП определяется как направлением электромагнитного поля ППП, так и спектральными зависимостями диэлектрических проницаемостей материалов МПК; в частности, оптические потери в пленке золота, поддерживающей распространение ППП, увеличиваются в коротковолновой части спектра. В результате в спектре отражения доминирующим оказывается минимум, сдвигающийся в длинноволновую область при уменьшении $\varphi$.

Магнитный контраст, определяемый соотношением (4), имеет особенности, зависящие от азимутального угла только в области резонансного возбуждения ППП, т.е при длине волны около $850 \mathrm{~nm}$ (рис. $2, b$ ) и около $650 \mathrm{~nm}$ для $s$-поляризации пробного излучения (рис. $3, b$ ). Во втором случае следует рассмотреть наличие двух видов близких по частоте ППП, распространяющихся под разными углами к направлению периодичности структуры и имеющих разные знаки проекции волновых векторов на напряженность приложенного магнитного поля и соответственно проявляющих противоположные знаки магнитооптических эффектов. Взаимодействие этих двух плазмонных возбуждений, каждое из которых имеет собственный спектр магнитооптического эффекта, приводит, по-видимому, к 
наблюдающейся знакопеременной зависимости магнитного контраста.

Следует также отметить, что для $s$-поляризации магнитооптический эффект по модулю в несколько раз меньше по сравнению со случаем $p$-поляризации зондирующего излучения. Это различие связано с более сильным рассогласованием направлений электрических полей ППП и падающей волны для случая $s$-поляризация света, чем для случая $p$-поляризации. Еще раз отметим, что для однородной пленки пермаллоя магнитооптический эффект не наблюдается для $s$-поляризации света.

\section{5. Заключение}

Таким образом, разработан и изучен новый тип МПК, образованного одномерной диэлектрической дифракционной решеткой с нанесенными на нее слоями благородного металла (золото) толщиной $80 \mathrm{~nm}$ и ферромагнитного сплава (пермаллой) толщиной $10 \mathrm{~nm}$. Было показано наличие характерных для МПК оптических и магнитооптических свойств, связанных с резонансным возбуждением ППП. В частности, спектры коэффициента отражения МПК показывают возбуждение ППП, соответствующим условиям фазового квазисинхронизма (2). Возбуждение ППП позволяет наблюдать в ферромагнитной пленке всего в $10 \mathrm{~nm}$ линейный поперечный магнитооптический эффект Керра с магнитным контрастом, достигающим 0.2 процента для $p$-поляризованного падающего света. В $s$-поляризованном излучении обнаружена смена знака магнитного контраста в области резонансного возбуждения ППП.

\section{Благодарности}

Образцы плазмонных кристаллов изготовлены с использованием материально-технической базы ЦКП НОЦ „Функциональные Микро/Наносистемы“ МГТУ им. Н.Э. Баумана (ID 74300).

\section{Финансирование работы}

Работа выполнена при финансовой поддержке РФФИ, проект № 19-02-00826.

\section{Конфликт интересов}

Авторы заявляют, что у них нет конфликта интересов.

\section{Список литературы}

[1] Maier S.A. Plasmonics: fundamentals and applications. Springer Science \& Business Media, 2007.

[2] Inoue M., Arai K., Fujii T., Abe M. // J. Appl. Phys. 1999. V. 85. N 8. P. 5768

[3] Khanikaev A.B., Baryshev A.B., Lim P.B., Uchida H., Inoue M., Zhdanov A.G., Fedyanin A.A., Maydykovskiy A.I., Aktsipetrov O.A. // Phys. Rev. B. 2008. V. 78. P. 193102.
[4] Ushioda S., Sasaki Y. // Phys. Rev. B. 1983. V. 27. P. 1401.

[5] González-Díaz J.B., García-Martín A., Armelles G., GarcíaMartín J.M., Clavero C., Cebollada A., Lukaszew R.A., Skuza J.R., Kumah D.P., Clarke R. // Phys. Rev. B. 2007. V. 76. N 15. P. 153402.

[6] Ferreiro-Vila E., Bendana X.M., Gonzalez-Diaz J.B., GarciaMartin A., Garcia-Martin J.M., Cebollada A., Armelles G., Meneses D., Munoz Sandoval E. // IEEE Trans. Magn. 2008. V. 44. N 11. P. 3303.

[7] Barnes W.L., Dereux A., Ebbesen T.W. // Nature. 2003. V. 424. № 6950. P. 824.

[8] Kretschmann E., Raether H. // Z. Naturforsch. A. 1968. V. 23. N 12. P. 2135.

[9] Otto A. // Zeitschrift fur Physik A Hadrons and nuclei. 1968. V. 216, N 4. P. 398.

[10] Bonanni V., Bonetti S., Pakizeh T., Pirzadeh Z., Chen J., Nogues J., Vavassori P., Hillenbrand R., Akerman J., Dmitriev A. // Nano Lett. 2011. V. 11. N 12. P. 5333.

[11] Grunin A.A., Zhdanov A.G., Ezhov A.A., Ganshina E.A., Fedyanin A.A. // Appl. Phys. Lett. 2010. V. 97. N 26. P. 261908.

[12] Belotelov V.I., Akimov I.A., Pohl M., Kotov V.A., Kasture S., Vengurlekar A.S., Achanta Venu Gopal, Yakovlev D.R., Zvezdin A.K., Bayer M. // Nat. Nanotechnol. 2011. V. 6. N 6. P. 370.

[13] Chekhov A.L., Krutyanskiy V.L., Ketsko V.A., Stognij A.I., Murzina T.V. // Opt. Mater. Express. 2015. V. 5. N 7. P. 1647.

[14] Rodionov I.A., Baburin A.S., Gabidullin A.R., Maklakov S.S., Peters S., Ryzhikov I.A., Andriyash A.V. // Sci. Rep. 2019. V. 9. N 1. P. 1.

[15] Belotelov V.I., Bykov D.A., Doskolovich L.L., Kalish A.N., Zvezdin A.K. // J. Opt. Soc. Am. B. 2009. V. 26. N 8. P. 1594.

[16] Borovkova O.V., Hashim H., Kozhaev M.A., Dagesyan S.A., Chakravarty A., Levy M., Belotelov V.I. // Appl. Phys. Lett. 2018. V. 112. N 6. P. 063101.

[17] Caballero B., Garcia-Martin A., Cuevas J.C. // ACS Photonics. 2016. V. 3. N 2. P. 203.

[18] Shaimanov A.N., Orlikovsky N.A., Khabushev E.M., Zverev A.V., Pishimova A.A., Sharonov G.V., Yankovskii G.M., Rodionov I.A., Baryshev A.V. // Photonics Nanostructures: Fundam. Appl. 2018. V. 32. P. 1. 